\title{
Switching on flowers: transient LEAFY induction reveals novel aspects of the regulation of reproductive development in Arabidopsis
}

\author{
Doris Wagner $^{1 *}$ and Elliot M. Meyerowitz ${ }^{2}$ \\ ${ }^{1}$ Department of Biology, University of Pennsylvania, Philadelphia, PA, USA \\ 2 Division of Biology, California Institute of Technology, Pasadena, CA, USA
}

\section{Edited by:}

Elena M. Kramer, Harvard University, USA

\section{Reviewed by:}

Pablo Daniel Jenik, Franklin and

Marshall College, USA

Levi Yant, Harvard University, USA

*Correspondence:

Doris Wagner, $103 \mathrm{G}$ Lynch

Laboratories, Department of Biology,

University of Pennsylvania, 415S.

University Avenue, Philadelphia, PA 19104, USA.

e-mail:wagnerdo@sas.upenn.edu
Developmental fate decisions in cell populations fundamentally depend on at least two parameters: a signal that is perceived by the cell and the intrinsic ability of the cell to respond to the signal. The same regulatory logic holds for phase transitions in the life cycle of an organism, for example the switch to reproductive development in flowering plants. Here we have tested the response of the monocarpic plant species Arabidopsis thaliana to a signal that directs flower formation, the plant-specific transcription factor LEAFY (LFY). Using transient steroid-dependent LEAFY (LFY) activation in Ify null mutant Arabidopsis plants, we show that the plant's competence to respond to the LFY signal changes during development. Very early in the life cycle, the plant is not competent to respond to the signal. Subsequently, transient LFY activation can direct primordia at the flanks of the shoot apical meristem to adopt a floral fate. Finally, the plants acquire competence to initiate the flower-patterning program in response to transient LFY activation. Similar to a perennial life strategy, we did not observe reprogramming of all primordia after perception of the transient signal, instead only a small number of meristems responded, followed by reversion to the prior developmental program. The ability to initiate flower formation and to direct flower patterning in response to transient LFY upregulation was dependent on the known direct LFY target APETALA1 (AP1). Prolonged LFY or activation could alter the developmental gradient and bypass the requirement for AP1. Prolonged high AP1 levels, in turn, can also alter the plants' competence. Our findings shed light on how plants can fine-tune important phase transitions and developmental responses.

Keywords: LEAFY, flower development, reproductive competence

\section{INTRODUCTION}

The entire above ground body of higher plants is generated postembryonically by a group of stem cells located in the shoot apical meristem (Steeves and Sussex, 1989). Lateral organs arise from primordia that form in the organogenic region at the periphery of the shoot apical meristem (Ha et al., 2010). During the Arabidopsis life cycle, different types of lateral organs are produced by these primordia (Steeves and Sussex, 1989; Poethig, 2003; Albani and Coupland, 2010). First, juvenile and adult rosette leaves are formed with axillary inflorescences in their axils. After bolting, the primordia give rise to cauline leaves subtending secondary inflorescence branches. Both axillary and secondary inflorescences repeat the body plan of the central shoot or primary inflorescence.

After production of a defined number of secondary inflorescences, there is an abrupt and irreversible switch to formation of flowers. Flowers are morphologically distinct from the lateral organs formed up to this point; they are determinate structures comprised of modified leaves arranged in four concentric rings or whorls: the protective sepals, the showy petals, the male reproductive structures, the stamens, and the female reproductive structures, the carpels (Poethig, 2003; Krizek and Fletcher, 2005; McKim and Hay, 2010). Two transcription factors, the plant-specific helix-turn-helix transcription factor LFY and the MADS box transcription factor APETALA1 (AP1) are critical for flower formation (Blazquez et al., 2006; Liu et al., 2009a; Moyroud et al., 2010). AP1 expression is both directly and indirectly induced by LFY and - in addition - is upregulated by at least two LFY independent pathways (Ruiz-Garcia et al., 1997; Parcy et al., 1998; Wagner et al., 1999; Abe et al., 2005; Wigge et al., 2005; Yamaguchi et al., 2009; Xu et al., 2010; Pastore et al., 2011). LFY has a second, later, role in flower development where it directly activates the floral homeotic genes that specify petal, stamen, and carpel identity (Busch et al., 1999; Wagner et al., 1999; Lamb et al., 2002; Krizek and Fletcher, 2005; Liu and Mara, 2010; McKim and Hay, 2010; Winter et al., 2011). AP1 contributes to this function indirectly (Liu et al., 2009b; Kaufmann et al., 2010; Winter et al., 2011).

We employed a steroid hormone-inducible LFY activation system to test the phenotypic consequences of transient LFY activation at different timepoints during development and in different mutant backgrounds. Our findings reveal that the plant's competence to respond to the LFY signal increases with increasing plant age, that this change in competence changes occurs in the absence of either LFY or AP1, but that LFY and AP1 can modulate 
the competence of the plant. In addition, we show that individual meristems respond to the stimulus, a scenario reminiscent of the regulation of reproductive development in perennial plants.

\section{MATERIALS AND METHODS}

Plant materials were in the Landsberg erecta genotype and grown on $1 / 2$ MS medium in continuous light (to be consistent with previous experiments; Wagner et al., 2004) until after dexamethasone treatment. Stratification at $4^{\circ} \mathrm{C}$ was for 7 days. The ap1-1, lfy-6, and 35S:LFY-GR genotypes have been previously described (Mandel et al., 1992; Weigel et al., 1992; Wagner et al., 1999). Several different types of steroid-mediated $35 \mathrm{~S}<$ LFY-GR activation in lfy-6, ap1-1, or lfy-6 ap1-1 mutants were performed. All treatments used dexamethasone (Sigma) in $0.1 \%$ ethanol. For transient activation plants were treated with $5 \mu \mathrm{M}$ dexamethasone for $2 \mathrm{~h}$, followed by rinsing of the plants with water. Prolonged dexamethasone treatments consisted of (a) daily applications of $0.5 \mu \mathrm{M}$ dexamethasone for 7 days (Figure 3), (b) a one-time application of $5 \mu \mathrm{M}$ dexamethasone without rinsing of the plants (Figure 4), or (c) daily applications of $5 \mu \mathrm{M}$ dexamethasone 7 days (Figure 6). After dexamethasone treatment, plants were transferred to soil and grown in continuous light. Phenotypes were scored after plants had bolted.

\section{RESULTS}

\section{DISTINCT PLANT RESPONSES TO EARLY AND LATE PLUSES OF LFY ACTIVITY}

Early in development (before day 5), activation of 35S:LFY-GR (Wagner et al., 1999) in lfy-6 null mutant seedlings by a single 2-h treatment with the synthetic steroid dexamethasone did not trigger any morphological change in the plants, while later in development the majority of the plants were responsive to the increased LFY activity (Figure 1). On average three to four axillary meristems switched from inflorescence to floral fate upon perception of the LFY signal (Figure 1A). Activation at a later timepoint during seedling development resulted in primordium fate alteration in a comparably narrow zone along the shoot, but shifted the responding zone to later-arising primordia (Figure 1A). Axillary meristems above and below those affected by the steroid treatment generated $l f y-6$ - like secondary inflorescences (Figure 1B). From day five or day seven onward meristems that would normally give rise to axillary or secondary inflorescence branches, respectively, instead gave rise to flowers (Figure 1C). Thus, transient LFY activation was not sufficient to switch the entire plant to reproductive development. Instead, individual axillary meristems - presumably only those which directly experienced the LFY signal-adopted floral fate. It is known that pre-existing primordia can be reprogrammed to adopt floral fate (Hempel and Feldman, 1995). Since the shoot apex on average has three very young primordia associated with it and initiates primordia at a rate of 1.9 per day (Smyth et al., 1990), we estimate the effective LFY pulse lasted at most $12 \mathrm{~h}$ for re-specification of the fate of four primordia.

We observed a second differential response to the transient LFY signal that changed with plant age (Figure 2). Early steroid treatment (day 5-8) reprogrammed primordia that would have given rise to axillary or basal secondary inflorescences to give



rise to flowers (Figures 1 and 2). However, these flowers, like lfy- 6 mutant flowers, consisted of sepals and unfused or fused carpels (Figure 2A). Later LFY activation (day 8-11) instead converted secondary inflorescences to flowers, which displayed partial rescue of the lfy- 6 floral homeotic defects (Figures 2B,E). When seedlings were treated after day 11 , primordia that would have given rise to the upper secondary inflorescences and to lfy-6 mutant flowers instead produced fully rescued wild-type flowers (Figures 2D,E). The different responses are probably not due to the type of primordium receiving the LFY stimulus, because cauline leaf/secondary inflorescence primordia can produce lfy null mutant, partially rescued, or wild-type flowers (Figures 2B,C). Rather, these results suggest that the competence of the meristem to respond to transient LFY activation by triggering flower-patterning differs early and later in the life cycle. Early in development, plants exhibit low competence, which supports the formation of determinate structures (lfy-6 flowers). Higher competence is required for development of normal flowers, this was only achieved at a later stage in the life cycle.

\section{EARLY AND LATE LFY ACTIVITIES REOUIRE AP1}

To test what role AP1 plays in the plant's response to a transient LFY signal, we introduced LFY-GR into the strong ap1-1 mutant background and into ap 1-1 lfy-6 double mutants. Single steroid treatments in the ap1-1 LFY-GR background caused primordia to convert from inflorescence to flower fate only rarely (in one of seven lines tested) and only when LFY was activated very late in development (Figures 3A,C). No increased conversion of the axillary or of the first two secondary inflorescences to flowers was observed even when we increased steroid hormone exposure to 

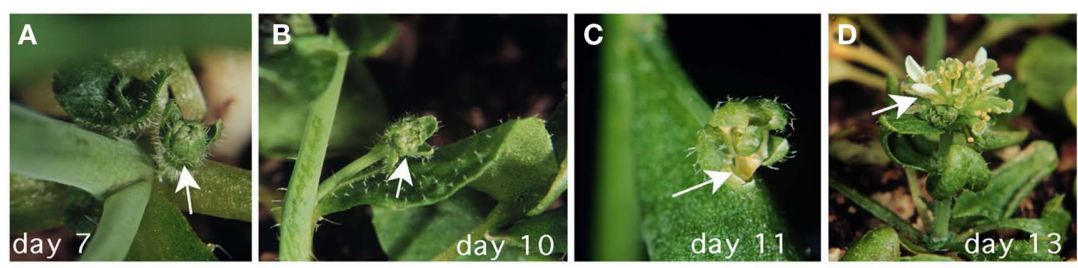

E

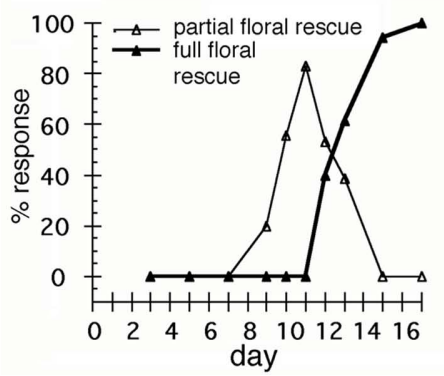

FIGURE 2 | Effect of transient LFY activation on floral organ identity. Ify- 6 35S:LFY-GR plants were treated as described in Figure 1 on day 7 (A), day 10 (B), day 11 (C), and day 13 (D). The axillary (A) or secondary (B-D) flowers that formed in response to dexamethasone treatment were examined for rescue of the Ify- 6 floral homeotic defects. Activation on day 7 resulted in formation of Ify- 6 flowers (A). Treatment on day 10 or day 11 caused partial rescue of the floral homeotic defects $(\mathbf{B}, \mathbf{C})$, while treatment on day 13 resulted in full rescue of the floral homeotic defects of Ify- 6 flowers. (E) The ratio of plants exhibiting a given response over the total number of plants per treatment day (\% response) was determined. For each treatment day 20-30 plants were scored after bolting. Ify-6 35S:LFY-GR plants were assayed for partial floral rescue $(\rightarrow$ ) or full floral rescue (fertile flowers) $(\longrightarrow)$.
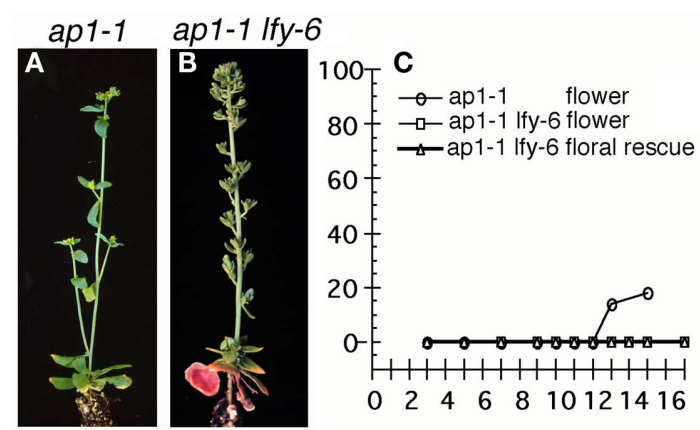

FIGURE 3 |Transient LFY activation in ap1-1 mutants. ap1-1 35SLFY-GR seedlings (A) or ap 1-1 Ify-6 35SLFY-GR seedlings (B) treated as described in Figure 1. Very few inflorescence to flower conversions were observed. (C) The ratio of plants exhibiting a given response over the total number of plants per treatment day (\% response). For each treatment day 20-30 plants were scored after bolting. ap 1-1 plants were scored for inflorescence to flower conversion (- - ). ap 1-1 Ify-6 plants were scored for inflorescence to flower conversion $(\square \square)$ and floral rescue $(\longrightarrow \mathbf{\Delta})$.

4 days (day 7-11; Figure 4) in ap1-1 mutants. By contrast, in the wild-type and in the $l f y$ - 6 genetic background, the 4-day induction caused conversion of all primordia to floral fate (Figures $4 \mathrm{~A}-\mathrm{C}$ ). Thus, in the absence of AP1, the plant's competence to respond to the LFY signal by specifying floral fate is much reduced. At later stages in development (increased competence) LFY alone was sufficient to induce floral fate (Figure 3C).

In ap 1-1 lfy-6 35S:LFY-GR plants none of the LFY-GR inductions tested [single steroid pulse (Figures 3B,C) or 4-day LFY activation (Figure 4D)] resulted in an alteration in the primordium
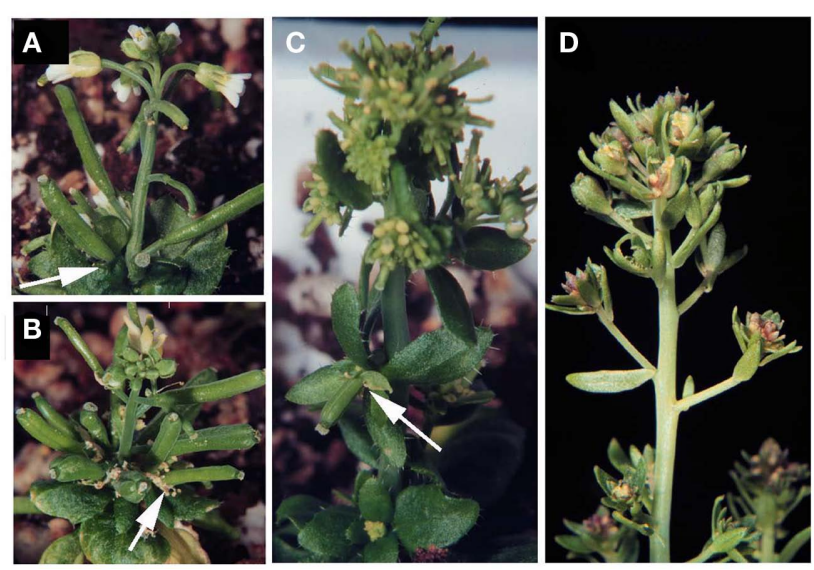

FIGURE 4 | Effect of prolonged LFY activation. Seedlings were treated once with $5 \mu \mathrm{M}$ dexamethasone on day 7, followed by further incubation for 4 days. Genotypes employed are wild-type (Ler) (A), Ify-6 (B), ap 1-1 (C), and ap 1-1 Ify-6 (D). White arrows point to basal secondary inflorescences converted into flowers $(\mathbf{A}, \mathbf{B})$, or a later-arising secondary inflorescence converted into a flower (C). ap 1-1 Ify-6 plants did not respond to this steroid induction (D).

fate, suggesting that apl lfy mutants are either unable to acquire increased competence to respond to the LFY signal, or acquire it much later than lfy or apl single mutants.

\section{PROLONGED LFY AND AP1 ACTIVITY ALTER MERISTEM COMPETENCE}

More continuous activation of LFY by daily treatment of seedlings from day 3 to day 10 with a tenth of the single-treatment dose caused transformation of axillary inflorescences to rescued flowers 


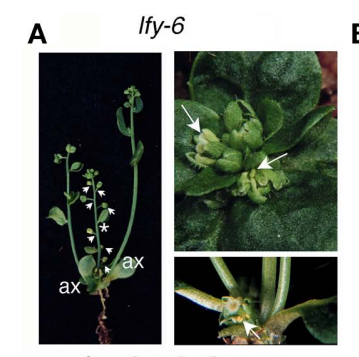

day $3-10 ; 0.5 \mu \mathrm{M}$



FIGURE 5 | Effect of prolonged LFY activation of meristem identity. Seedlings were treated for 7 days daily starting at day 3 with $0.5 \mu \mathrm{M}$ dexamethasone. Phenotypes of all plants were observed at maturity. (A) Left panel. A mature Ify- 6 LFY-GR plant. Many axillary and all secondary inflorescences have been converted into flowers. One of these flowers still has some inflorescence traits and is subtended by a bract (asterisk). Many axillary inflorescences produce rescued, fertile flowers (data not shown). Later formed axillary meristems produce Ify-6-like inflorescences (ax). Right panel top. Secondary inflorescences in a Ify- 6 LFY-GR plant were converted to single flowers subtended by a cauline leaves. These flowers are partially rescued because petaloid (arrow) and stamenoid organs are formed. Right panel bottom. A flower with stamens formed instead of an axillary inflorescence in a Ify-6 LFY-GR plant (arrow). (B) Plants treated at day 10, day 13 , and day 14 by a single $2 \mathrm{~h} 5 \mu \mathrm{M}$ dexamethasone application were compared to plants treated for 7 days with $0.5 \mu \mathrm{M}$ dexamethasone starting at day 3. The number of inflorescences above the last inflorescence converted to a flower were counted for at least 20 plants per treatment day. (C) A Ify-6 LFY-GR plant in a weak $35 \mathrm{~S}<\mathrm{AP} 1$ background. Plants were treated once at day 7 with $5 \mu \mathrm{M}$ dexamethasone as described in Figure 1 The arrowhead points to a partially rescued flower.

(Figures 5A,B). Thus, prolonged activation of LFY results in "late" LFY activity at early stages. These findings suggest that prolonged presence of LFY can increase the competence of early arising primordia and commit the primary shoot to flower production. This does not appear to be simply due to the amount of LFY protein present in the nucleus because the total dexamethasone dose in the continuous treatment (Figure 5B) was less than that of a single treatment. In addition, increasing the hormone concentration for single treatments did not give a qualitative change in the response (data not shown). Moreover, the prolonged LFY signal irreversibly directed all primordia on the central stem to adopt a flower fate (Figures 5A,B).

Furthermore, precocious presence of AP1 (in a weak $A P 1$ overexpression line; in lfy-6 35S $<$ AP1 $35 \mathrm{~S}<$ LFY-GR plants) also altered the competence of early arising primordia such that a single transient activation of LFY at day 5 or day 7 now resulted in conversion of axillary inflorescences to rescued wild-type looking flowers (Figure 5C). Continuous presence of AP1 or prolonged LFY activation could thus alter the plant's competence to respond to LFY.

Finally, early prolonged and strong activation of LFY (Wagner et al., 2004) triggered conversion of the entire primary inflorescence into a single flower, which formed stamens in all genotypes tested (Figure 6). This suggests that prolonged strongly elevated LFY levels can overcome most of the competence requirements we observed in response to transient or less strong prolonged LFY signals (Figures 3C and 4D).
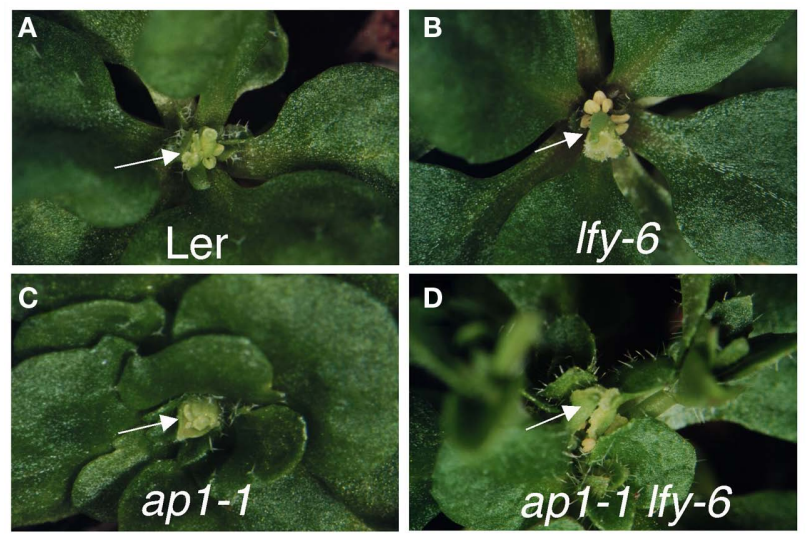

FIGURE 6 | The effect of prolonged early strong activation of LFY. Plants of the genotypes indicated were treated daily from day 3 to day 10 with $5 \mu \mathrm{M}$ dexamethasone. In all cases (A-D), the entire primary inflorescence was converted into a flower. The single terminal flower (white arrow) displayed at least partial rescue of the floral homeotic defects present in Ify null flowers (B,D).

\section{DISCUSSION}

Here we describe a temporal change in the plant's response to transient LFY activation from unresponsive, to ability to specify floral fate, and finally ability to direct flower patterning. The plant acquired the increased competence to respond to the LFY signal in the absence of LFY. Competence to trigger flower formation was acquired more slowly in the ap 1 mutant background, with a delay of at least 9 days. This is unlikely to be due to AP1-mediated changes in the plant prior to LFY induction, since in lfy mutants AP1 expression is induced only at or after day 11 (Pastore et al., 2011), while lfy mutant plants displayed increased ability to specify floral fate relative to apl lfy plants prior to this timepoint. Instead, it highlights a role for AP1 as a direct LFY target and LFY co-factor in specification of floral fate and in flower patterning (Weigel and Nilsson, 1995; Wagner et al., 1999; Liu et al., 2009b). That AP1 can alter the plants' competence was demonstrated by the ability of weak $35<\mathrm{AP} 1$ plants to condition all lfy mutant primordia - even those formed very early in development - to convert to wild-type flowers.

Early in development transiently elevated LFY requires AP1 activity to induce floral fate. Later in development inductive requirements are less stringent, allowing LFY alone to induce floral fate. In addition, prolonged high LFY activity drastically affects the developmental gradient, allowing "late activity" early and independent of AP1. Under extreme inductive conditions and late in development a monocarpic plant -like Arabidopsis thus seems to default to reproductive development. These findings are consistent with previous observations reported for the plant's response to constitutively elevated $L F Y$ levels (Weigel and Nilsson, 1995). Our studies using transient LFY activation allowed more detailed insight into the temporal changes in the competence of the plants to respond to LFY and further revealed that transient inductive signals do not reprogram the entire shoot. Instead individual primordia respond to the signal suggesting that monocarpic 
and perennial life strategies may share more features than previously suspected (Albani and Coupland, 2010; Bergonzi and Albani, 2011).

The distinct effects of a single, transient activation of LFY at different stages in the life cycle may be due to a developmental gradient created through increasing levels of an activator or decreasing levels of an inhibitor, which determine the competence of primordia to respond to LFY. This increased competence affects later arising - more acropetal - primordia on the main stem as well as axillary branches, which grow out during the period of increased competence. Possible candidates for this signal are components of the age-sensing pathway (mir156, mir172, and their target genes) or changes in the chromatin state (Poethig, 2009; Amasino, 2010; Bergonzi and Albani, 2011). Indeed, 35S:mir156 plants have phenotypic defects similar to those described for Arabidopsis mutants that have a more perennial growth habit (Melzer et al., 2008; Yamaguchi et al., 2009), while the expression of many genes that direct floral fate and flower development are known to be subject to chromatin repression prior to the correct developmental stage (Lafos and Schubert, 2009; Zheng and Chen, 2011).

Recently, direct LFY targets were identified genomewide (Moyroud et al., 2011; Winter et al., 2011). Although these studies were not conducted under conditions where LFY was present

\section{REFERENCES}

Abe, M., Kobayashi, Y., Yamamoto, S., Daimon, Y., Yamaguchi, A., Ikeda, Y., Ichinoki, H., Notaguchi, M., Goto, K., and Araki, T. (2005). FD, a bZIP protein mediating signals from the floral pathway integrator FT at the shoot apex. Science 309, 1052-1056.

Albani, M. C., and Coupland, G. (2010). Comparative analysis of flowering in annual and perennial plants. Curr. Top. Dev. Biol. 91, 323-348.

Amasino, R. (2010). Seasonal and developmental timing of flowering. Plant J. 61, 1001-1013.

Bergonzi, S., and Albani, M. C. (2011). Reproductive competence from an annual and a perennial perspective. J. Exp. Bot. 62, 4415-4422.

Blazquez, M. A., Ferrandiz, C., Madueno, F., and Parcy, F. (2006). How floral meristems are built. Plant Mol. Biol. 60, 855-870.

Busch, M. A., Bomblies, K., and Weigel, D. (1999). Activation of a floral homeotic gene in Arabidopsis. Science $285,585-587$.

Ha, C. M., Jun, J. H., and Fletcher, J. C. (2010). Shoot apical meristem form and function. Curr. Top. Dev. Biol. 91, 103-140.

Hempel, F. D., and Feldman, L. J. (1995). Specification of chimeric flowering shoots in wild-type Arabidopsis. Plant J. 8, 725-731.

Kaufmann, K., Wellmer, F., Muino, J. M., Ferrier, T., Wuest, S. E., Kumar, V., Serrano-Mislata, A., Madueno, F., Krajewski, P., Meyerowitz, E. M., Angenent, G. C., and Riechmann, J.

only transiently, they nevertheless revealed developmental stagedependent differences in LFY activity; LFY ability to associate with target gene regulatory regions and to regulate target gene expression was dependent on the developmental context (Winter et al., 2011). In summary, flower formation and development is controlled in at least two ways. First, by transcriptional upregulation of LFY (Blazquez et al., 2006; Moyroud et al., 2010) and second, by independently controlling the response of the plant to the LFY signal. These two parameters are not simply additive, they interact. When the duration of the inductive signal is short, plant intrinsic parameters dictate the result, that is to say the competence state of the plant is critical. When the signal is prolonged, it can override plant intrinsic requirements. Integrating these interactions transforms a simple switch into a rheostat to allow maximum control of a vital developmental transition.

\section{ACKNOWLEDGMENTS}

We thank Ayako Yamaguchi and Scott Poethig from comments on this manuscript. This work was supported by National Science Foundation grant IOS-0849298 to Doris Wagner and Department of Energy Office of Basic Energy Sciences, Division of Chemical Sciences, Geosciences, and Biosciences grant DE-FG02-88ER13873 to Elliot M. Meyerowitz.

(2008). Flowering-time genes modulate meristem determinacy and growth form in Arabidopsis thaliana. Nat. Genet. 40, 1489-1492.

Moyroud, E., Kusters, E., Monniaux, M., Koes, R., and Parcy, F. (2010). LEAFY blossoms. Trends Plant Sci. 15, 346-352.

Moyroud, E., Minguet, E. G., Ott, F., Yant, L., Pose, D., Monniaux, M., Blanchet, S., Bastien, O., Thevenon, E., Weigel, D., Schmid, M., and Parcy, F. (2011). Prediction of regulatory interactions from genome sequences using a biophysical model for the arabidopsis LEAFY transcription factor. Plant cell 23, 1293-1306.

Parcy, F., Nilsson, O., Busch, M. A., Lee, I., and Weigel, D. (1998). A genetic framework for floral patterning. Nature 395, 561-566.

Pastore, J. J., Limpuangthip, A., Yamaguchi, N., Wu, M. F., Sang, Y., Han, S. K., Malaspina, L., Chavdaroff, N., Yamaguchi, A., and Wagner, D. (2011). LATE MERISTEM IDENTITY2 acts together with LEAFY to activate APETALA1. Development 138, 3189-3198.

Poethig, R. S. (2003). Phase change and the regulation of developmental timing in plants. Science 301, 334-336.

Poethig, R. S. (2009). Small RNAs and developmental timing in plants. Curr. Opin. Genet. Dev. 19, 374-378.

Ruiz-Garcia, L., Madueno, F., Wilkinson, M., Haughn, G., Salinas, J., and
Martinez-Zapater, J. M. (1997). Different roles of flowering-time genes in the activation of floral initiation genes in Arabidopsis. Plant Cell 9, 1921-1934.

Smyth, D. R., Bowman, J. L., and Meyerowitz, E. M. (1990). Early flower development in Arabidopsis. Plant Cell 2, 755-767.

Steeves, T. A., and Sussex, I. (1989). Pattern in Plant Development. Cambridge: Cambridge University Press.

Wagner, D., Sablowski, R. W., and Meyerowitz, E. M. (1999). Transcriptional activation of APETALA1 by LEAFY. Science 285, 582-584.

Wagner, D., Wellmer, F., Dilks, K. William, D., Smith, M. R., Kumar, P. P., Riechmann, J. L., Greenland, A. J., and Meyerowitz, E. M. (2004). Floral induction in tissue culture: a system for the analysis of leafy-dependent gene regulation. Plant J. 39, 273-282.

Weigel, D., Alvarez, J., Smyth, D. R., Yanofsky, M. F., and Meyerowitz, E. M. (1992). LEAFY controls floral meristem identity in Arabidopsis. Cell 69, 843-859.

Weigel, D., and Nilsson, O. (1995). A developmental switch sufficient for flower initiation in diverse plants. Nature 377, 495-500.

Wigge, P. A., Kim, M. C., Jaeger, K. E., Busch, W., Schmid, M., Lohmann, J. U., and Weigel, D. (2005). Integration of spatial and temporal information during floral induction in Arabidopsis. Science 309, 1056-1059. 
Winter, C. M., Austin, R. S., BlanvillainBaufume, S., Reback, M. A., Monniaux, M., Wu, M. F., Sang, Y., Yamaguchi, A., Yamaguchi, N., Parker, J. E., Parcy, F., Jensen, S. T., Li, H., and Wagner, D. (2011). Leafy target genes reveal floral regulatory logic, cis motifs, and a link to biotic stimulus response. Dev. Cell 20, 430-443.

Xu, M., Hu, T., McKim, S. M., Murmu, J., Haughn, G. W., and Hepworth, S. R. (2010). Arabidopsis BLADEON-PETIOLE 1 and 2 promote floral meristem fate and determinacy in a previously undefined pathway targeting APETALA1 and AGAMOUSLIKE24. Plant J. 63, 974-989.

Yamaguchi, A., Wu, M. F., Yang, L., Wu, G., Poethig, R. S., and Wagner, D. (2009). The microRNAregulated SBP-Box transcription factor SPL3 is a direct upstream activator of LEAFY, FRUITFULL, and APETALA1. Dev. Cell 17, 268-278.

Zheng, B., and Chen, X. (2011). Dynamics of histone $\mathrm{H} 3$ lysine 27 trimethylation in plant development. Curr. Opin. Plant Biol. 14, 123-129.
Conflict of Interest Statement: The authors declare that the research was conducted in the absence of any commercial or financial relationships that could be construed as a potential conflict of interest.

Received: 29 August 2011; paper pending published: 06 September 2011; accepted: 13 September 2011; published online: 11 October 2011.

Citation: Wagner D and Meyerowitz EM (2011) Switching on flowers: transient LEAFY induction reveals novel aspects of the regulation of reproductive development in Arabidopsis. Front. Plant Sci. 2:60. doi: 10.3389/fpls.2011.00060

This article was submitted to Frontiers in Plant Evolution and Development, a specialty of Frontiers in Plant Science. Copyright (c) 2011 Wagner and Meyerowitz. This is an open-access article subject to a non-exclusive license between the authors and Frontiers Media $S A$, which permits use, distribution and reproduction in other forums, provided the original authors and source are credited and other Frontiers conditions are complied with. 